\title{
(2) OPEN ACCESS \\ Association between suicide risk and traumatic brain injury in adults: a population based cohort study
}

\author{
Yueh-Chien Lu, ${ }^{1}$ Ming-Kung Wu, ${ }^{2}$ Li Zhang, ${ }^{3}$ Cong-Liang Zhang, ${ }^{4}$ Ying-Yi Lu, ${ }^{5,6,7}$ \\ Chieh-Hsin Wu (D) ${ }^{8,9}$
}

For numbered affiliations see end of article.

\section{Correspondence to}

Dr Chieh-Hsin Wu, Kaohsiung, Taiwan; wujoeys@gmail.com

$\mathrm{Y}-\mathrm{CL}$ and $\mathrm{M}-\mathrm{KW}$ contributed equally.

LZ and C-LZ contributed equally.

Received 14 June 2019 Revised 1 December 2019 Accepted 31 December 2019

Published Online First 3 February 2020

\section{Check for updates}

(c) Author(s) (or their employer(s)) 2020. Re-use permitted under CC BY-NC. No commercial re-use. See rights and permissions. Published by BMJ.

To cite: Lu Y-C, Wu M-K,

Zhang L, et al.

Postgrad Med J

2020:96:747-752

\section{ABSTRACT}

Background Traumatic brain injury (TBI) is a major cause of death and disability worldwide, and its treatment is potentially a heavy economic burden. Suicide is another global public health problem and the second leading cause of death in young adults. Patients with TBI are known to have higher than normal rates of non-fatal deliberate self-harm, suicide and all-cause mortality. The aim of this study was to explore the association between TBI and suicide risk in a Chinese cohort.

Method This study analysed data contained in the Taiwan National Health Insurance Research Database for 17504 subjects with TBI and for 70016 subjects without TBI matched for age and gender at a ratio of 1 to 4. Cox proportional hazard regression analysis was used to estimate subsequent suicide attempts in the TBI group. Probability of attempted suicide was determined by Kaplan-Meier method.

Results The overall risk of suicide attempts was 2.23 times higher in the TBI group compared with the non-TBI group (0.98 vs 0.29 per 1000 person-years, respectively) after adjustment for covariates. Regardless of gender, age or comorbidity, the TBI group tended to have more suicide attempts, and the risk attempted suicide increased with the severity of TBI. Depression and alcohol attributed disease also increased the risk of attempted suicide in the TBI group.

Conclusion Suicide is preventable if risk factors are recognised. Hence, TBI patients require special attention to minimise their risk of attempted suicide.

\section{INTRODUCTION}

Traumatic brain injury (TBI), which is defined as damage to the skull or the brain and its frameworks through an external force, ${ }^{1}$ is a major cause of death and disability worldwide, and its treatment is potentially a heavy economic burden. The number of TBIs that occur annually worldwide is an estimated 1.5 to 2 million; the global prevalence is $12 \%$ to $16.7 \%$ in males and $8.5 \%$ in females, respectively. In Taiwan, up to $25 \%$ of the approximately 52000 annual cases of TBI are fatal. ${ }^{2}$ The WHO projects that TBI will be the third leading cause of death or disability by $2020 .^{3}$ Traumatic brain injury is most common in young adults and the elderly. Mild TBI is far more common than severe TBI. The severity of various consequences of TBI is known to increase proportionally to the severity of the TBI; typical consequences include physical disability, cognitive impairments and mood disorders.
Suicide is a global public health problem. Approximately 800000 people commit suicide every year, and suicide is the second leading cause of death in young adults. In Taiwan, the number of deaths by suicide in 2016 was 3765, and the standardised mortality rate was 12.3 persons per population of $100000 .^{4}$ According to statistics released by the Taiwan Ministry of Health and Welfare, suicide is the $12^{\text {th }}$ most common cause of death. Suicide occurs throughout the life span and across all socioeconomic strata. Moreover, suicide has a potentially immense socioeconomic burden on individuals, families, communities and nations. ${ }^{5}$

The causes of suicide are multifactorial. Individuals with TBI have higher rates of nonfatal deliberate self-harm, suicide and all-cause mortality compared with the general population. ${ }^{67}$ Although recent studies suggest that TBI might be an important risk factor for suicide, studies of the association between TBI severity and suicide risk have reported conflicting results between countries and races. Simpson ${ }^{8}$ and Fonda et $a l^{9}$ reported TBI severity contributed no meaningful differences to the risk of suicide attempts in the study of Australian population or US veterans who served in either Iraq or Afghanistan, respectively; whereas Madsen et $\mathrm{al}^{7}$ showed the risk of suicide was higher for TBI individuals with evidence of structural brain injury in Denmark.

However, most studies investigating the relation of suicide and TBI have some shortcomings in methodology, such as small clinical samples, especially very small number of TBI cases with suicide and self-reported data. Besides, little population-based data for this association are available. In the Simpson's survey, they only included small number of patients $(n=172)$ in Australia. In the Fonda's analysis, only US veterans were included. The majority of military veterans were young (mean age $=28.7$ years) male (84\%). The definition of TBI severity was based on self-reported duration of consciousness loss, alternation of consciousness and posttraumatic amnesia, not on objective examinations. Besides, moderate (6\%) to severe (6\%) TBI subsample cases were too small to bias the estimates.

In Denmark, Madsen et al conducted a retrospective cohort study (1977 to 2014) and showed that a statistically significant increased suicide was present in TBI individuals. However, their research results were potentially biassed because they did not include all mild TBI patients treated prior to 1995. Also, there were no available records on what treatment TBI patients received, making it difficult to 
determine the TBI severity. Additionally, this study was performed in population of the Western world, not those of Eastern Asia. Generalising data retrieved from a study of a relatively homogeneous and wealthy Scandinavian people is not persuasive. Finally, large-scale population-based studies for the relationship between TBI severity and suicide risk in Asian cohorts are scarce. Therefore, this study used the Taiwan National Health Insurance Research Database (NHIRD) to clarify the association between TBI and subsequent occurrence of suicide as well as the effect of TBI severity in Chinese cohorts. In our analysis, the TBI severity is based on objective codes or examinations and then correlation is estimated through adjusting for all important suicide risks.

\section{METHODS}

\section{Database}

Data retrieved from the NHIRD maintained by the Taiwan healthcare system was used in this population-based cohort study. The encrypted NHIRD contains medical data of more than $99 \%$ of the 23.74 million Taiwan residents. The Taiwan national health insurance programme allowed researchers to access this administrative database for patients. The NHIRD contains enrolment information for all patients and comprehensive data for their use of healthcare resources. In accordance with the Personal Electronic Data Protection Law of Taiwan, any information that can be used to identify beneficiaries and hospitals must be removed from the NHIRD. This cohort study analysed 1996 to 2010 data from the Longitudinal Health Insurance Database 2010, which is an NHIRD subset that collects data of 1 million randomlysampled beneficiaries from the primary NHIRD. This study identified and classified diseases according to the diagnostic codes in the International Classification of Diseases, Ninth Revision, Clinical Modification (ICD-9-CM).

\section{Study population}

The study included 17504 patients who were older than 20 years and had been diagnosed with TBI (ICD-9-CM code 800, 803 to 804 and 850 to 854 ; operation codes $01.23,01.24$, $01.25,01.31,01.39$ and 02.01 ) during 1996 to $2010 .^{10}$ The analysis was limited to patients who had received $\geq 1$ diagnoses during inpatient care or $\geq 2$ TBI diagnoses during ambulatory visits in order to ensure data accuracy. The date of the first clinical visit for TBI was defined as the index date. The TBI cohort excluded patients who had been diagnosed with any mental disorder (ICD-9-CM code 290 to 319) before the index date. The TBI cases were further classified as severe, moderate or mild. Severe TBI was defined as a TBI that required surgery in the course of inpatient treatment; moderate TBI was defined as a TBI that required hospitalisation but not surgery; mild TBI was defined as a TBI that did not require inpatient treatment. ${ }^{11}$ The ICD-9-CM, E-Codes for suicide attempts (950 to 959 and 980 to 989$)^{12}$ were assigned by psychiatrists. Patients were excluded if they had a ICD-9 code for suicide attempt before the index date. Additionally, 70016 patients without TBI were identified to enhance the power of statistical tests in stratified analysis. The non-TBI patients were randomly selected from the registry of beneficiaries. Patients were eligible for inclusion in the non-TBI group if they had not been diagnosed with TBI during 1996 to 2010 and had no history of suicide attempts before enrolment. The non-TBI group and the TBI group were matched at a 4:1 ratio for gender, age and index year (year of TBI diagnosis). Figure 1 shows the workflow of the study procedure.

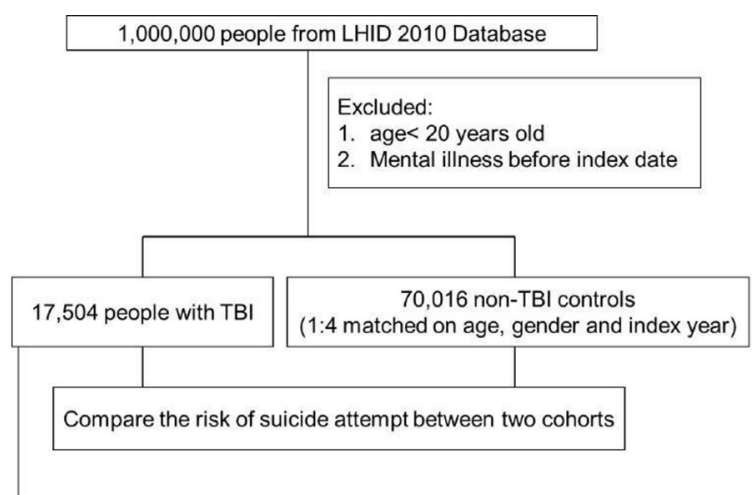

Examine the effect of TBI severity on the risk of suicide attempt

Figure 1 Flow diagram summarising the process of enrolment. LHID,Longitudinal Health Insurance Database; TBI, traumaticbrain injury.

\section{Outcome and comorbidities}

The outcome was any occurrence of suicide attempts during the follow-up period. Both cohorts were followed until 31 December 2010 or until the occurrence of suicide attempts.

A review of the psychiatry literature reveals that very few modifiable risk factors have been clearly defined. ${ }^{13}$ Therefore, most studies have focused on largely unmodifiable risk factors such as age and gender and on risk factors that are common physical comorbidities, such as hypertension (ICD-9-CM codes 401 to 405 ), hyperlipidaemia (ICD-9-CM code 272), congestive heart failure (ICD-9-CM code 428), diabetes mellitus (ICD-9-CM code 250), coronary artery disease (ICD-9-CM code 410 to 414), liver cirrhosis (ICD-9-CM code 571), depression (ICD-9-CM code 296.2 to 296.3, 300.4 and 311), tobacco use disorder (ICD-9-CM code 350.1) and alcohol attributed disease (ICD-9-CM codes 291.0 to 9, 303, 305.0, 357.5, 425.5, 535.3, 571.0 to $3,980.0$ and $\mathrm{V} 11.3)^{13}$ were potential confounders in this analysis because they are unhealthy lifestyle behaviours associated with both TBI and mental illness. ${ }^{11}$

For each participant, urbanisation index and income-related insurance payment amounts were used as proxy measures of socioeconomic status at follow-up. Urbanisation index was categorised into three groups: high (metropolises), medium (small cities and suburban areas) and low (rural areas). ${ }^{14}$ Insurance premiums were categorised into three groups according to the monthly insurance payment by the enrollee: NT\$0 to 20000 ; NT\$20000 to 40000 and more than NT\$40000. ${ }^{15}$

\section{Statistical analyses}

We used $\mathrm{X}^{2}$ test to compare clinical characteristics and distributions of categorical demographics between the TBI and nonTBI cohorts. The Wilcoxon rank-sum test and Student's t-test were used to compare mean age and follow-up time (y) between the two cohorts, as appropriate. The Kaplan-Meier analysis was used to estimate cumulative incidence of suicide attempts, and the differences between the curves were compared by two-tailed log-rank test. For TBI patients, survival was calculated until an ambulatory visit for suicide attempts, hospitalisation or the end of the study period (31 December 2010), whichever came first. Incidence rates of suicide attempts estimated in 1000 personyears were compared between the two cohorts. Cox proportional hazard regression models were used to investigate the HR and 95\% CI for suicide attempts for individuals with TBI if the proportional hazards assumption was satisfied. Multivariable 
Table 1 Baseline characteristics of patients with and without traumatic brain injury

\begin{tabular}{|c|c|c|c|}
\hline \multirow[b]{2}{*}{ Variables } & \multicolumn{2}{|c|}{ Traumatic brain injury } & \multirow[b]{2}{*}{ P value } \\
\hline & Yes $(n=17504)$ & No $(n=70016)$ & \\
\hline $\begin{array}{l}\text { Mean age at enrolment } \\
\text { (years, SD) }\end{array}$ & $44.2(16.9)$ & $44.4(16.8)$ & 0.058 \\
\hline \multicolumn{4}{|l|}{ Age group, $\mathbf{n}(\%)$} \\
\hline $20-49$ & 11489 (65.64) & $45956(65.64)$ & \\
\hline$\geq 50$ & 6015 (34.36) & $24060(34.36)$ & 1.000 \\
\hline \multicolumn{4}{|l|}{ Gender, n (\%) } \\
\hline Men & $9916(56.65)$ & 39664 (56.65) & \\
\hline Women & $7588(43.35)$ & 30352 (43.35) & 1.000 \\
\hline \multicolumn{4}{|l|}{ Income, n (\%) } \\
\hline Low $(<\mathrm{NT} \$ 20000)$ & $14051(80.27)$ & $55662(79.50)$ & \\
\hline $\begin{array}{l}\text { Medium (NT\$20000-39 } \\
\text { 999) }\end{array}$ & $2454(14.02)$ & $9141(13.06)$ & \\
\hline High (>NT\$40 000) & $999(5.71)$ & $5213(7.45)$ & $<0.001$ \\
\hline \multicolumn{4}{|l|}{$\begin{array}{l}\text { Degree of urbanisation, } \\
n(\%)\end{array}$} \\
\hline Urban & $10649(60.84)$ & 41044 (58.62) & \\
\hline Suburban & $5767(32.95)$ & $23245(33.20)$ & \\
\hline Rural & $1088(6.22)$ & 5727 (8.18) & $<0.001$ \\
\hline \multicolumn{4}{|l|}{ Comorbidity, n (\%) } \\
\hline Hypertension & $6905(39.45)$ & $16956(24.22)$ & $<0.001$ \\
\hline Diabetes mellitus & $4243(24.24)$ & $10143(14.49)$ & $<0.001$ \\
\hline Hyperlipidaemia & $5799(33.13)$ & $16083(22.97)$ & $<0.001$ \\
\hline Coronary artery disease & $1269(7.25)$ & $1990(2.84)$ & $<0.001$ \\
\hline Congestive heart failure & $1330(7.60)$ & $2371(3.39)$ & $<0.001$ \\
\hline Liver cirrhosis & $6042(34.52)$ & $16654(23.79)$ & $<0.001$ \\
\hline Depression & $1753(10.01)$ & $5185(7.41)$ & $<0.001$ \\
\hline Tobacco use disorder & $176(1.01)$ & $433(0.62)$ & $<0.001$ \\
\hline Alcohol attributed disease & $1375(7.86)$ & $1682(2.40)$ & $<0.001$ \\
\hline $\begin{array}{l}\text { Newly diagnosed } \\
\text { suicide attempts, n (\%) }\end{array}$ & $154(0.88)$ & $314(0.45)$ & $<0.001$ \\
\hline $\begin{array}{l}\text { Period of developing } \\
\text { suicide attempt, years } \\
\text { (median, IQR) }\end{array}$ & 4.2 (1.5 to 7.0$)$ & $\begin{array}{l}8.0(4.9 \text { to } \\
11.1)\end{array}$ & $<0.001$ \\
\hline
\end{tabular}

Cox models were adjusted for gender, age, income, urbanisation and relevant comorbidities. A two-tailed $\mathrm{p}$ value less than 0.05 was considered statistically significant. Statistical Analysis Software, V.9.4, was used for all data analyses (SAS Institute, Cary, North Carolina, USA).

\section{RESULTS}

\section{Baseline characteristics of the TBI and non-TBI cohorts}

Table 1 presents the baseline demographic characteristics and comorbidities in the two cohorts. In the TBI cohort, $43.35 \%$ patients were female. Compared with the non-TBI cohort, the TBI cohort had significantly higher percentages of patients with hypertension ( 39.45 vs $24.22 ; \mathrm{p}<0.001)$, diabetes mellitus (24.24 vs $14.49 ; \mathrm{p}<0.001)$, hyperlipidaemia $(33.13$ vs 22.97 ; $\mathrm{p}<0.001)$, coronary artery disease $(7.25$ vs $2.84 ; \mathrm{p}<0.001)$, congestive heart failure (7.60 vs 3.39; $\mathrm{p}<0.001)$, liver cirrhosis (34.52 vs $23.79 ; \mathrm{p}<0.001$ ), tobacco use disorder (1.01 vs 0.62 ; $\mathrm{p}<0.001)$, depression (10.1 vs $7.41 ; \mathrm{p}<0.001)$ and alcohol attributed disease $(7.86$ vs $2.40 ; \mathrm{p}<0.001)$. During a median observation time of 4.2 years, $0.88 \%$ (154) of the TBI patients had suicide attempt ( $\mathrm{IQR}=1.5$ to 7.0$)$. The incidence of suicide attempt in the TBI cohort was significantly $(\mathrm{p}<0.001)$ higher than that in the non-TBI cohort (314 suicide attempts out of 70016 age-matched and gender-matched controls $(0.45 \%)$ during a median observation time of 8.0 years $(\mathrm{IQR}=4.9$ to 11.1)). The median duration of follow-up for suicide attempts was significantly shorter in the TBI group (4.2 years) compared with the non-TBI group (8.0 years).

\section{Incidence and risk of suicide attempts}

In table 2, the incidence and HR for suicide attempts are stratified by gender, age and comorbidity. During the follow-up period, the overall risk of suicide attempt was 2.23 times higher in the TBI group compared with the non-TBI group (0.98 vs 0.29 per 1000 person-years, respectively) after adjustment for gender, age and related comorbidities (diabetes mellitus, hyperlipidaemia, hypertension, coronary artery disease, congestive heart failure, liver cirrhosis, depression, alcohol attributed disease and tobacco use disorder).

Table 2 Incidence and HR of suicide attempt by demographic characteristics, comorbidity and follow-up duration among patients with or without TBI

\begin{tabular}{|c|c|c|c|c|c|c|}
\hline \multirow[b]{2}{*}{ Variables } & \multicolumn{2}{|l|}{ Patients with TBI } & \multicolumn{2}{|c|}{ Patients without TBI } & \multicolumn{2}{|c|}{ Compared with non-TBI } \\
\hline & Suicide attempts & Rate & Suicide attempts & Rate & Crude $\mathrm{HR}^{*}(95 \% \mathrm{Cl})$ & Adjusted HR* $(95 \% \mathrm{Cl})$ \\
\hline Overall & 154 & 0.98 & 314 & 0.29 & $3.31(2.54$ to 4.32$) \dagger$ & 2.23 (1.81 to 2.76$) \dagger$ \\
\hline \multicolumn{7}{|l|}{ Gender } \\
\hline Men & 83 & 0.92 & 181 & 0.30 & $3.36(2.30$ to 4.89$) \dagger$ & 2.04 (1.53 to 2.72$) \dagger$ \\
\hline Women & 71 & 1.05 & 133 & 0.29 & $3.27(2.24$ to 4.76$) \dagger$ & $2.49(1.82$ to 3.41$) \dagger$ \\
\hline \multicolumn{7}{|c|}{ Stratify by age } \\
\hline $20-49$ & 117 & 1.07 & 202 & 0.29 & $3.56(2.83$ to 4.49$) \dagger$ & $2.40(1.88$ to 3.07$) \dagger$ \\
\hline$\geq 50$ & 37 & 0.77 & 112 & 0.31 & $2.45(1.68$ to 3.56$) \dagger$ & 1.85 (1.27 to 2.72$) \ddagger$ \\
\hline \multicolumn{7}{|c|}{ Comorbidity§ } \\
\hline No & 16 & 0.30 & 91 & 0.16 & 1.82 (1.07 to 3.09$)$ ๆ & $1.77(1.04 \text { to } 3.02)^{* *}$ \\
\hline Yes & 138 & 1.32 & 223 & 0.45 & $2.85(2.29$ to 3.54$) \dagger$ & $2.20(1.75$ to 2.77$) \dagger$ \\
\hline
\end{tabular}

*Model adjusted for age, gender, income, urbanisation level and relevant comorbidities (hypertension, diabetes mellitus, hyperlipidaemia, coronary artery disease, congestive heart failure, liver cirrhosis, depression, tobacco use disorder and alcohol attributed disease).

$\mathrm{tp}<0.001$;

$\ddagger \mathrm{P}=0.002$.

§atients with any examined comorbidities, including hypertension, diabetes mellitus, hyperlipidaemia, coronary artery disease, congestive heart failure, liver cirrhosis, depression, tobacco use disorder and alcohol attributed disease, were classified as the comorbidity group.

Ip $=0.028 ; * * \mathrm{P}=0.035$.

Rate, incidence rate in per 1000 person-years; TBI, traumatic brain injury. 


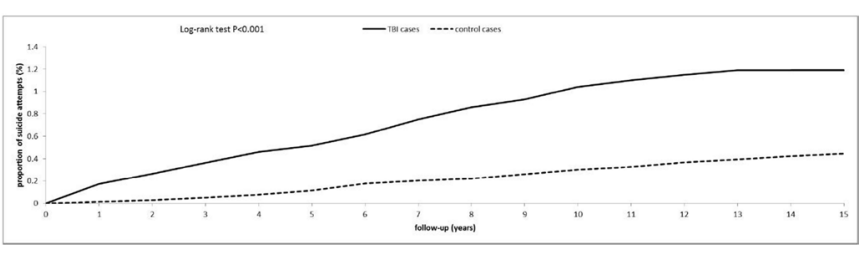

Figure 2 Cumulative incidence of suicide attempts among patients with traumatic brain injury and the control cohort. TBI,traumatic brain injury.

Gender-specific analyses showed that, in the TBI group, the incidence of suicide attempts was higher in women than in men (1.05 vs 0.92 per 1000 person-years, respectively). Additionally, in comparison with the non-TBI group, the TBI group had a significantly higher risk of suicide attempts in both genders (adjusted HR $=2.49,95 \%$ CI 1.82 to 3.41 for women; adjusted $\mathrm{HR}=2.04,95 \% \mathrm{CI} 1.53$ to 2.72 for men).

In age-specific risk comparisons of the two cohorts, the TBI cohort consistently showed a significantly higher risk of attempted suicide in all age groups. Regardless of comorbidities, the risk of suicide attempt was higher in TBI group than non-TBI group. Notably, the risk of attempted suicide was even higher in TBI patients who had comorbidities.

Figure 2 compares the Kaplan-Meier curves for the cumulative incidence of suicide attempts between the TBI and nonTBI groups at the 15 year follow-up. The Kaplan-Meier curves showed a significantly higher cumulative incidence of suicide attempts in the TBI group compared with the non-TBI group (log-rank test $\mathrm{p}<0.001)$.

\section{Risks factors for suicide attempts in TBI patients}

The Cox regression analysis revealed two risk factors for suicide attempts in the TBI group: depression (adjusted $\mathrm{HR}=5.73$, 95\% CI 4.14 to 7.93 ) and alcohol attributed disease (adjusted $\mathrm{HR}=3.29,95 \% \mathrm{CI} 2.30$ to 4.72 ). However, the risk of attempted suicide in this group decreased as age increased (table 3 ).

\section{Risk of suicide attempt and TBI severity}

Table 4 shows that all patients with TBI had a higher than normal risk of suicide attempts. Additionally, the risk of attempted suicide increased as the severity of TBI increased.

\section{DISCUSSION}

To our knowledge, this is the first large-scale population-based study to investigate the association between TBI and subsequent suicide risk in a Chinese population. The TBI group in this study had a 2.23-fold higher risk of suicide attempts compared with the non-TBI group (0.98 vs 0.29 per 1000 person-years,

Table 3 Cox regression model: significant predictors of suicide attempt after traumatic brain injury

\begin{tabular}{llr}
\hline Variables & Adjusted $\mathrm{HR}^{*}(95 \% \mathrm{Cl})$ & P value \\
\hline Depression & $5.73(4.14$ to 7.93$)$ & $<0.001$ \\
Alcohol attributed disease & $3.29(2.30$ to 4.72$)$ & $<0.001$ \\
Older age & $0.83(0.74$ to 0.94$)$ & 0.002 \\
\hline
\end{tabular}

The adjusted $\mathrm{HR}$ and $95 \% \mathrm{Cl}$ were estimated by a stepwise Cox proportional hazards regression method.

* Model adjusted for age, gender, income, urbanisation level and relevant comorbidities (hypertension, diabetes mellitus, hyperlipidaemia, coronary artery disease, congestive heart failure, liver cirrhosis, depression, tobacco use disorder and alcohol attributed disease) respectively). Regardless of age, gender or comorbidity, all TBI subgroups had a higher than normal risk of attempted suicide. Moreover, patients with severe TBI had a slightly greater risk of attempted suicide compared with those with mild TBI. In the TBI group, the strongest risk factors for attempted suicide were depression and alcohol attributed disease.

This study had several notable advantages. First, this population-based study analysed longitudinal data contained in an insurance claims database over a long observation period (15 years) and in a large population (1000000 Taiwan residents). The analyses were adjusted for time-varying factors that are well recognised as major risk factors for suicide. Therefore, recall bias was limited compared with analyses based on self-reported data for suicide behaviour used in other surveys. Second, the Taiwan National Health Insurance programme includes over 99.5\% residents in Taiwan. Therefore, the observed association between TBI and suicide was representative of the actual population, and the possibility of selection bias was minimal.

However, this study has several limitations. First, the insurance claims database used in this analysis did not include data for suicidal ideation or completed suicide. Therefore, the analysis could only consider attempted suicide. Second, this insurance claims database did not include individuals who did not seek medical care because their TBI or self-harm behaviours were mild. Therefore, potential bias in identification may have resulted in an underestimated incidence of attempted suicide. Third, this study did not consider chronic pain, which has a high incidence in TBI patients and has a significant association with depression. ${ }^{16}$ Moreover, our conclusions were based on analysis of secondary data from an insurance claims database. Unexpected biasses from the effect of different numbers and severity of concomitant comorbidities were possible. Data were unavailable for potential confounding factors such as family history of suicide or child abuse or for environmental factors such as stressful life events or prolonged stress. Additionally, this study could only analyse diagnoses of alcohol attributed illness and tobacco use disorder rather than habitual use of alcohol or tobacco. Finally, this study did not consider social factors such as marital status and social support and did not consider patient characteristics such as Glasgow Coma Scale score, trauma mechanisms and duration of lost consciousness.

Previous studies have investigated the prevalence of suicidal ideation, attempted suicide and death by suicide in TBI patients in the community. ${ }^{17}$ Systemic review found robust evidence of the relationship between TBI and elevated risk of suicide. ${ }^{18}$ A TBI can cause dysfunction of the frontal lobes and frontalsubcortical circuits, then resulting in aggression, poor decisionmaking and impulsivity. Impulsivity and aggression also have a strong association with suicidal behaviour. ${ }^{19}$ This study revealed a markedly higher cumulative incidence of suicide attempts in the TBI cohort compared with the non-TBI cohort and the TBI group had a 2.23-fold higher risk of attempted suicide compared with the non-TBI group in Taiwan population. These results are consistent with a longitudinal follow-up study by Lauren et $a l^{20}$ who reported that TBI is a major risk factor for suicidal behaviour. Moreover, data collected using standardised selfreported measures of post-traumatic stress disorder, depression, suicidal thoughts and behaviours in military personnel also indicates that suicide risk is higher in subjects with a history of multiple TBIs compared with those with only one or no TBI. ${ }^{21} 22$

In the general population, risk factors for attempted suicide include gender, age and history of previous suicide attempts. ${ }^{23} \mathrm{~A}$ notable gender difference is that the rate of attempted suicide is higher in females whereas the rate of completed suicides is higher 
Table 4 Incidence and HRs for suicide attempt stratified by the severity of TBI

\begin{tabular}{|c|c|c|c|c|c|}
\hline Variables & $\mathrm{N}$ & Suicide attempts & Rate & Crude $\mathrm{HR}^{*}(95 \% \mathrm{Cl})$ & Adjusted HR* $(95 \% \mathrm{Cl})$ \\
\hline Without TBI & 70016 & 314 & 0.29 & 1.00 (reference) & 1.00 (reference) \\
\hline Mild TBI & 5319 & 37 & 0.93 & $3.06(2.16$ to 4.33$) \dagger$ & $2.22(1.56$ to 3.17$) \dagger$ \\
\hline Moderate TBI & 11661 & 111 & 0.98 & $3.21(2.57$ to 3.99$) \dagger$ & $2.23(1.77$ to 2.82$) \dagger$ \\
\hline Severe TBI & 524 & 6 & 1.35 & $4.44(1.98$ to 9.97$) \dagger$ & $2.32(1.03$ to 5.26$) \neq$ \\
\hline
\end{tabular}

Rate, incidence rate in per 1000 person-years.

*Model adjusted for age, gender, income, urbanisation level and relevant comorbidities (hypertension, diabetes mellitus, hyperlipidaemia, coronary artery disease, congestive heart failure, liver cirrhosis, depression, tobacco use disorder and alcohol attributed disease).

$t p<0.001 ; \neq p=0.044$.

$\mathrm{TBI}$, traumatic brain injury.

in males. ${ }^{24}$ In the current study, both male and female patients in the TBI group had a higher risk of attempted suicide compared with their counterparts in the non-TBI group. Specifically, males in the TBI group had 2.04-fold higher risk of attempted suicide; females in the TBI group had a 2.49-fold higher risk of attempted suicide. In Teasdale et $a l^{6}{ }^{6}$ an analysis of a Denmark cohort similarly reported that females with TBI had a higher suicide mortality rate compared with males with TBI. Hartkopp et $a l^{25}$ speculated that females have more difficulties bearing the consequence of injury because the role of physical attractiveness in self-image is more important in women compared with men.

The psychiatric disorder most commonly (50\% to 60\%) implicated in death by suicide is depression. ${ }^{26}$ Anxiety, chronic pain and drug abuse are other independent risk factors for attempted suicide. $^{27}$ Psychotherapeutic interventions implemented early (within the first year) after injury may reduce the progression of major depression in TBI. ${ }^{28}$ In our study, depression and alcohol attributed disease are significantly associated with suicide after TBI (adjusted HR 5.73, 95\% CI 4.14 to 7.93 , p < 0.001 ; adjusted HR $3.29,95 \%$ CI 2.30 to $4.72, \mathrm{p}<0.001$, respectively).

\section{Main messages}

Patients in Chinese ancestry initially diagnosed with traumatic brain injury (TBI) have a high risk of developing suicide attempts.

- The risk of suicide attempts is increased associated with the severity of TBI.

- The increased risk of suicide is less in TBI patients aged above 50 years; thus, older age may protect TBI cohort from suicide attempts.

\section{Current research questions}

Does the TBI severity correlate with suicide attempts in Chinese patients?

- Would increased exposure to traumatic brain injury earlier in life increase the risk of suicide attempts?

- Do older TBI patients have higher suicide attempts risks?

\section{What is already known on the subject}

- Both traumatic brain injury and suicide are worldwide health problems and burdens.

- Patients with TBI are known to have higher than normal mortality.
In the elderly, the most common cause of TBI is falls. Elderly patients with TBI also have significantly worse outcomes for mortality and functional impairment. ${ }^{29}$ Age is a major factor in functional decline after $\mathrm{TBI}^{30}$ A previous population-based study revealed the incidence of suicide was lower in TBI patients younger than 21 years or older than 60 years. ${ }^{6}$ Table 3 shows that, after stratification by age, the risk of suicide attempt in elderly patients was significantly lower in the current study (adjusted HR 0.83 ; 95\% CI 0.74 to $0.94 ; \mathrm{p}=0.002$ ). A possible explanation for the age difference is that elderly patients have less cognitive and physical capability to carry out a suicide after injury.

The association between TBI severity and suicide has also attracted the interest. The relative risk of attempted suicide is three to four times higher in patients with severe TBI compared with the general population. ${ }^{17}$ Clinical evidence also indicates that both severe and mild TBI are associated with increased suicidal tendencies. ${ }^{31}$ Brenner et $a l^{32}$ reported that compared with those without TBI, death by suicide was 1.34 -fold higher in military veterans with a history of severe TBI, while veterans with a history of relative mild TBI, that is, fracture or contusion, had a 1.98 times higher risk of attempted suicide. Pain may have contributed to the increased risk of suicide in the mild TBI group. In comparison, increased suicide risk by severity of TBI in our study revealed that patients with mild, moderate and severe TBI had 2.22-fold, 2.23-fold and 2.32-fold, respectively, higher risks of attempted suicide compared with patients without TBI (table 4).

\section{CONCLUSION}

The worldwide consensus is that suicide is a preventable cause of death. The findings of our study suggest that depression, alcoholic attributed disease and high severity of TBI may increase the risk of attempted suicide in patients with TBI. These factors serve as vital mechanisms through which TBI influence suicide attempts. Suicide attempts are strong indicators for death of suicide. ${ }^{33}$ Suicide prevention requires a cooperative effort by society, the family and the individual. The results of this study suggested that intensive interventions for identifying individuals with a high risk of suicidal behaviour can have large and positive public health effect. Thereby, future research is warranted to identify other mechanisms of these association, including possible biological interaction of TBI and other predictors such as severity to elucidate whether prompt intervention for TBI cases could reduce this risk.

\section{Author affiliations}

${ }^{1}$ Department of Family Medicine, Kaohsiung Chang Gung Memorial Hospital and Chang Gung University College of Medicine, Kaohsiung, Taiwan

${ }^{2}$ Department of Psychiatry, Kaohsiung Chang Gung Memorial Hospital and Chang Gung University College of Medicine, Kaohsiung, Taiwan 
${ }^{3}$ Department of Neurosurgery, The Affiliated Zhangjiagang Hospital of Soochow University, Zhangjiagang, China

${ }^{4}$ Department of Cardiology, Hebei Quyang Renji Hospital, Quyang, China

${ }^{5}$ Department of Dermatology, Kaohsiung Veterans General Hospital, Kaohsiung, Taiwan

${ }^{6}$ Department of Nursing, Shu-Zen Junior College of Medicine and Management, Kaohsiung, Taiwan

${ }^{7}$ Graduate Institute of Medicine, College of Medicine, Kaohsiung Medical University, Kaohsiung, Taiwan

${ }^{8}$ Division of Neurosurgery, Department of Surgery, Kaohsiung Medical University Hospital, Kaohsiung, Taiwan

${ }^{9}$ Department of Surgery, School of Medicine, College of Medicine, Kaohsiung Medical University, Kaohsiung, Taiwan

Contributors Conceptualisation, M-KW; Data curation, Y-CL; Formal analysis, LZ; Investigation, LZ; Methodology, M-KW; Project administration, C-LZ; Resources, Y-YL; Software, Y-YL; Supervision, C-HW; Validation, C-LZ; Writing - original draft, Y-CL; Writing - review and editing, C-HW. Y-CL and M-KW were equal contributors in this study. LZ and C-LZ were equal contributors in this study.

Funding This work was supported by KMUH105- 5M17, KMUH1066T09,KMUH108-8M23 and KMU- Q108029 from Kaohsiung Medical University Hospital.

Competing interests None declared.

Patient consent for publication Not required.

Ethics approval In accordance with Declaration of Helsinki guidelines, the current study was performed and approved by the Institutional Review Board of Kaohsiung Medical University Hospital (KMUHIRB-EXEMPT(I)-20160021).

Provenance and peer review Not commissioned; externally peer reviewed.

Data availability statement All data relevant to the study are included in the article

Open access This is an open access article distributed in accordance with the Creative Commons Attribution Non Commercial (CC BY-NC 4.0) license, which permits others to distribute, remix, adapt, build upon this work non-commercially, and license their derivative works on different terms, provided the original work is properly cited, appropriate credit is given, any changes made indicated, and the use is non-commercial. See: http://creativecommons.org/licenses/by-nc/4.0/.

ORCID iD

Chieh-Hsin Wu http://orcid.org/0000-0001-5116-8004

\section{REFERENCES}

1 Menon DK, Schwab K, Wright DW, et al. Position statement: definition of traumatic brain injury. Arch Phys Med Rehabil 2010;91:1637-40.

2 Chiu W-T, Huang S-J, Tsai S-H, et al. The impact of time, legislation, and geography on the epidemiology of traumatic brain injury. J Clin Neurosci 2007;14:930-5.

3 Hyder AA, Wunderlich CA, Puvanachandra P, et al. The impact of traumatic brain injuries: a global perspective. NeuroRehabilitation 2007;22:341-53.

4 Lin H-T, Lai C-H, Perng H-J, et al. Insomnia as an independent predictor of suicide attempts: a nationwide population-based retrospective cohort study. BMC Psychiatry 2018;18:117.

5 Saxena S, Krug EG, Chestnov 0, et al. Preventing suicide : a global imperative. Geneva: World Health Organization, 2014

6 Teasdale TW, Engberg AW. Suicide after traumatic brain injury: a population study. J Neurol Neurosurg Psychiatry 2001;71:436-40.

7 Madsen T, Erlangsen A, Orlovska S, et al. Association between traumatic brain injury and risk of suicide. JAMA 2018;320:580-8.

8 Simpson G, Tate R. Suicidality after traumatic brain injury: demographic, injury and clinical correlates. Psychol Med 2002;32:687-97.
9 Fonda JR, Fredman L, Brogly SB, et al. Traumatic brain injury and attempted suicide among veterans of the wars in Iraq and Afghanistan. Am J Epidemiol 2017; 186:220-6.

10 Tung Y-C, Tu H-P, Tsai W-C, et al. Increased incidence of herpes zoster and postherpetic neuralgia in adult patients following traumatic brain injury: a nationwide populationbased study in Taiwan. PLoS One 2015;10:e0129043.

11 Chi Y-C, Wu H-L, Chu C-P, et al. Traumatic brain injury and affective disorder: a nationwide cohort study in Taiwan, 2000-2010. J Affect Disord 2016;191:56-61.

12 Wei H-T, Lan W-H, Hsu J-W, et al. Risk of suicide attempt among adolescents with conduct disorder: a longitudinal follow-up study. J Pediatr 2016;177:292-6.

13 Hu L-Y, Shen C-C, Hung J-H, et al. Risk of psychiatric disorders following symptomatic menopausal transition: a nationwide population-based retrospective cohort study. Medicine 2016:95:e2800.

14 Wu C-H, Tsai T-H, Su Y-F, et al. Traumatic brain injury and substance related disorder: a 10-year nationwide cohort study in Taiwan. Neural Plast 2016;2016:8030676.

15 Chung K-H, Li C-Y, Kuo S-Y, et al. Risk of psychiatric disorders in patients with chronic insomnia and sedative-hypnotic prescription: a nationwide population-based followup study. J Clin Sleep Med 2015;11:543-51.

16 Ruan X, Wu H, Wang D. Suicidal behaviour following traumatic brain injury. Brain Inj 2017;31:717-8.

17 Simpson G, Tate R. Suicidality in people surviving a traumatic brain injury: prevalence, risk factors and implications for clinical management. Brain Inj 2007;21:1335-51.

18 Bahraini NH, Simpson GK, Brenner LA, et al. Suicidal ideation and behaviours after traumatic brain injury: a systematic review. Brain Impairment 2013;14:92-112.

19 Gvion Y, Apter A. Aggression, impulsivity, and suicide behavior: a review of the literature. Arch Suicide Res 2011;15:93-112.

20 Fisher LB, Pedrelli P, Iverson GL, et al. Prevalence of suicidal behaviour following traumatic brain injury: longitudinal follow-up data from the NIDRR traumatic brain injury model systems. Brain Inj 2016;30:1311-8.

21 Wisco BE, Marx BP, Holowka DW, et al. Traumatic brain injury, PTSD, and current suicidal ideation among Iraq and Afghanistan U.S. veterans. J Trauma Stress 2014;27:244-8.

22 Bryan CJ, Clemans TA. Repetitive traumatic brain injury, psychological symptoms, and suicide risk in a clinical sample of deployed military personnel. JAMA Psychiatry 2013:70:686-91.

23 Turecki G, Brent DA. Suicide and suicidal behaviour. Lancet 2016;387:1227-39.

24 Gaynes BN, West SL, Ford CA, et al. Screening for suicide risk in adults: a summary of the evidence for the U.S. preventive services Task force. Ann Intern Med 2004;140:822-35.

25 Hartkopp A, Brønnum-Hansen H, Seidenschnur AM, et al. Suicide in a spinal cord injured population: its relation to functional status. Arch Phys Med Rehabil 1998;79:1356-61.

26 Hawton K, Casañas I Comabella C, Haw C, et al. Risk factors for suicide in individuals with depression: a systematic review. J Affect Disord 2013;147:17-28.

27 Zalsman G, Hawton K, Wasserman D, et al. Suicide prevention strategies revisited: 10-year systematic review. Lancet Psychiatry 2016;3:646-59.

28 Roy D, Koliatsos V, Vaishnavi S, et al. Risk factors for new-onset depression after firsttime traumatic brain injury. Psychosomatics 2018:59:47-57.

29 Krishnamoorthy V, Distelhorst JT, Vavilala MS, et al. Traumatic brain injury in the elderly: burden, risk factors, and prevention. J Trauma Nurs 2015;22:204-8. quiz E34

30 Kolakowsky-Hayner SA, Hammond FM, Wright J, et al. Ageing and traumatic brain injury: age, decline in function and level of assistance over the first 10 years postinjury. Brain Inj 2012;26:1328-37.

31 Silver JM, Kramer R, Greenwald S, et al. The association between head injuries and psychiatric disorders: findings from the new Haven NIMH epidemiologic catchment area study. Brain Inj 2001:15:935-45.

32 Brenner LA, Homaifar BY, Adler LE, et al. Suicidality and veterans with a history of traumatic brain injury: precipitants events, protective factors, and prevention strategies. Rehabil Psychol 2009;54:390-7.

33 Ougrin D, Tranah T, Stahl D, et al. Therapeutic interventions for suicide attempts and self-harm in adolescents: systematic review and meta-analysis. J Am Acad Child Adolesc Psychiatry 2015;54:97-107. 